\title{
OPERATIONAL TEST AND FIELD MEASUREMENT OF D-10-3 MAGNET
}

R. Yamada, H. Ishimoto, R.E. Pighetti and M.E. Price March 26, 1976

\begin{abstract}
A ten-foot D-type superconducting bending magnet ( $D-10-3$ ) was canned in a cryostat and tested using the pool boiling method. The quench behavior, AC Ioss, and field distribution were measured with a newly developed data acquisition system. Harmonic analysis of magnetic field was done and results are presented.
\end{abstract}




\section{Introduction}

A ten-foot superconducting $D$-series bending magnet (D-10-3) for the Energy Doubler project was canned into an originally designed cryostat and a laminated iron core was welded on the outside. The cross section of this magnet is shown in Fig. 1.

The structure of the cryostat was designed such that the coil was cooled with forced circulating subcooled liquid helium. Due to the unavailability of a forced circulating system for this magnet it was tested using the normal pool boiling method. Due to the structure of the cryostat, the magnet was mounted at a $25^{\circ}$ angle with the horizontal. Junction boxes, which were needed for heat leak measurements, were modified, and a warm bore for the field measurements was put in the beam pipe.

After the magnet was quenched many times, the field distribution, the AC loss, and the higher order harmonic components (mainly 4-pole and 6-pole) were measured. Previously the magnet coil without the can and the core was measured by the Energy Doubler group and our group. Its AC loss data is reported in TM-638. ${ }^{1}$ The superconducting wire of this magnet is MCA 17 strand unsoldered cable.

\section{Assembly and Cool Down}

The most difficult part of the assembly was splicing the superconductor from the magnet to the current leads. The primary problem was limited space for the splice. There was only about a 2 -inch long working space. Also the splicing resistance had to be very small.

The 23 strand soldered cable of the power leads and 17 strand unsoldered cable from the magnet were: covered with a copper 
sheath with a U-shape, crimped together tightly and then soldered. To prevent them from moving when excited, the two leads were bundled tightly with teflon insulation between them. The resistance of this connection was about $10^{-8} \Omega$ per joint.

In our test setup, the single phase space is connected with the two phase space through a specially installed $\mathrm{U}$ tube at the bottom. The $20 \mathrm{~K}$ shield $\mathrm{IN}$ and OUT lines are also connected in the same manner. While testing the magnet liquid nitrogen was put through the shield line instead of $20 \mathrm{~K}$ He gas. During cool down, we used liquid nitrogen to precool the magnet and then used liquid helium. The liquids were forced to pass through the two phase region and then the single-phase region for heat exchange mechanism. Cooling efficiency was quite good. These steps took only about 40 and 45 minutes respectively.

The heat load to the $4.2^{\circ} \mathrm{K}$ system was measured during operation with the helium level down below the copper leads, and with a liquid nitrogen shield. The evaporated gas was forced through a heat exchanger installed in a water tank, and then sent to a flow meter. The rough value from this measurement was $\sim 2.5 \mathrm{~W}$ at $4.2 \mathrm{~K}$, which is in good agreement with M. Kuchnir's more accurate $\operatorname{data}^{2}$.

\section{Training curve}

The most interesting to know is how high the field of the final form of a D series 10 foot magnet with canning and laminated iron core can go. The magnetic field inside the warm bore was measured using a $\mathrm{Hall}$ probe.

Quenching currents were measured first with long laminations 
(116"), which are shorter than the coil by $4 \frac{1}{2}$ inches at each end. Training curves are shown in Fig. 2. The maximum field obtained with the long iron laminations is about $36.4 \mathrm{kG}$, which is lower than we expected. We estimated that the highest field point occurs in the windings at the end, and is higher than the central field by $11 \%$.

Later we cut the laminations by an additional 7 inches at each end and tested again. The top curve in Fig. 2 corresponds to the data with short iron laminations. The maximum field went up a little bit up to $37.5 \mathrm{~kg}(\sim 3 \%)$.

To check the effect of the end laminations, the cut-out laminations were temporarily put back at one end and clamped. The quenching current decreased back to its original value with long iron laminations. The quench curve which was measured by the Energy Doubler group for D-10-3 without iron laminations, is also shown in Fig. 2. The calculated transfer constant of $8.07 \mathrm{G} / \mathrm{A}$ by S. Snowdon was used here.

The short sample data curves of the wire used and operation load lines of the D-10-3 magnet are shown in Fig. 3. Without iron, the central field had gone up to $32.3 \mathrm{kG}$, with the highest field of $41.4 \mathrm{kG}$ (with $28 \%$ enhancement) at the coil ends, and both of these points are represented by cross marks. It seems the current reached the short sample value in this case.

In our two cases with iron laminations the central field is represented by a solid line of $10.0 \mathrm{G} / \mathrm{A}$ and circled crosses. According to $S$. Snowdon, the calculated transfer constant is 10.2 G/A, which is in good agreement with ours. The top edges of the innermost conductors are represented by a broken line at 
11.1 G/A and by uncircled crosses.

The highest field value for the long iron was estimated from K. Koepke's measurements. This field occurs at the end winding. and is about $12 \%$ higher than the central field value for long iron. The high field point at the end winding for the short iron is estimated to be $3 \%$ higher than the central field. These two points are represented by triangles.

In both short and long iron cases, the highest field points are close to short sample data, but are low. There are many possible reasons for not reaching short sample data; for example, the relative movement of iron and coil at high field, poor cooling, etc. One good possibility is wire movement due to magnetic force at the end portion. Two triangular points corresponding to high field at end in Fig. 3, are on a would-be hyperbolic line, suggesting that the magnetic force at the ends is almost the same for the two cases with iron. 4. Quench Values vs. Ramp Rate and Temperature

The ramp rate dependence of the quenching current was also investigated. The results are given in Fig. 4. This flat dependence up to $10 \mathrm{kG} / \mathrm{sec}$ agrees well with that obtained without iron by the Energy Doubler group. This also may suggest the quenching is starting by mechanical movement due to magnetic force, not by any thermal effort.

The above measurements were made at $4.2 \mathrm{~K}$, but the operating temperature of the Energy Doubler magnets is expected to be 4.6 4.7 K. Heat transfer in the subcooled helium flow is quite different from that in the pool boiling helium, but it is useful to know the quenching currents in pool boiling helium at higher temperatures. 
For this purpose, the cryostat was pressurized. Fig. 5 shows the temperature dependence of the quench current, which is almost flat up to $4.7 \mathrm{~K}(\sim 23 \mathrm{psia})$. In this region, the values seem to be smaller than those estimated from the short sample limit as shown by two points at $4.2 \mathrm{~K}$. Above $4.7 \mathrm{~K}$, the quench current seems to follow a line, which decreases $19 \%$ for an increase of $1^{\circ} \mathrm{K}$ in operating temperature. This fact suggests that the magnet quenches due to the constant magnetic force up to $4.7 \mathrm{~K}$ and then due to short sample data at higher temperatures. 5. Quench Behavior

The behaviors of the current, the voltage and the resistance of the magnet after quench were investigated by using the on-line computer system. Also the energy dumped into the magnet and dump resistor were calculated.

The voltage from the shunt; that across the magnet, the inductive part of which is subtracted using a bucking coil; and that across the dump resistor are sent to the ADC's and PDPII. The details of the software will be described elsewhere. Using these data, the resistance of the magnet and the energies lost in the magnet and the dump resistor $(0.05 \Omega)$ are calculated instantly. Figs. $6,7,8$ and 9 are displays of these values, where the time scale is $55 \mathrm{msec} / \mathrm{div}$.

Usually $85 \sim 90 \%$ of the stored energy, which is about $100 \mathrm{~kJ}$ is dumped into the dump resistor outside the cryostat. Typically 10 to $15 \mathrm{~kJ}$ is lost in the magnet with the present setup. To know how the normal region propagates, the resistance of the magnet was plotted versus the energy lost in the coil as shown in Fig. 10. The behavior in Fig. 10 is quite similar to that 
obtained without iron by the Energy Doubler group.

During initial operations, the SCR firing circuit misfired on several occasions and a lot of energy was dumped accidentally into the magnet. Up to $73 \mathrm{~kJ}$ was dissipated in the magnet, but we were able to determine from later operation that no damage was done. The superconducting cabled wire is not soldered and it has only B-stage glass insulation. If it were soldered cabled wire we would have had beads from melten solder and possibly we might have done damage to the coil. This is a merit of unsoldered wire.

\section{AC Loss}

Recently we switched the data acquisition system from the DPO-PDPII ${ }^{3}$ to the CAMAC-PDPII system, so that we could get more accurate data. This system has I2-bit $A D$ converters and the signal is digitized to 5000 points during each ramp.

The results of $A C$ loss measurements on the magnet are shown in Figs. 11 and 12. The ramp rate dependence is very weak, and Iinear up to $\sim 5 \mathrm{kG} / \mathrm{sec}$. Its gradient is roughly proportional to $B_{\max }$, which is very reasonable. The dependence of the AC Ioss on the maximum field was measured for cases of both $0 \mathrm{kG}$ and $9 \mathrm{kG}$ starting fields. The field dependence curve starting from $0 \mathrm{kG}$ showed a break at around $30 \mathrm{kG}$, and then increased more sharply than below $30 \mathrm{kG}$. This behavior is very similar to that observed without iron ${ }^{1}$. As we suggested before, wire movement seems to occur at high fields. The data with iron is higher than that without iron. The difference is due to hysteresis losses in the laminations and due to eddy current losses in the metallic parts of the cryostat. 
7. Field Measurement with Hall Probe

Before running harmonic analysis, field measurements along the central axis were made to get a feeling for the actual field shape. Probe number HTR-0618 (made by F.W. Bell) was used. Fig. 13 shows the vertical field distributions along its axis with long and short iron laminations.

With long iron, the field decreases very smoothly at the end, and no bump was observed. But with short iron, a very small bump can be seen at the coil edge.

The remanent field distribution was measured along the center line and $7.5 \mathrm{~mm}$ above and below the center line, as shown in Fig. 14. The cause of the relative shift of these three lines is not clear. The remanent field value is typically 5 to 6 Gauss, but it seems to be changing and decreasing in the course of time. It has a prominent peak at the end, which is common to superconducting magnets. Well inside the magnet, the distribution has a periodic structure having a $3 "$ period. The cause of this periodicity is unknown.

\section{Harmonic Analysis of Magnetic Field}

One of the main purposes of this test was to measure the field quality of the final form of a 10' magnet. Measurements were made using both DC field and pulsed field. A block diagram of this equipment is shown in Fig. 15. The details of the measuring system and procedures will be described elsewhere.

The field distribution along the median plane of a dipole magnet can be expressed by the formula

$$
\begin{aligned}
& B_{y}(x)=B_{0}\left(1+b_{1} x+b_{2} x^{2}+b_{3} x^{3}+b_{4} x^{4}+\ldots\right) \\
& B_{x}(x)=B_{0}\left(a_{1} x+a_{2} x^{2}+a_{3} x^{3}+a_{4} x^{4}+\ldots\right)
\end{aligned}
$$


where $B_{0}$ is the central field, $b_{1}, b_{2}, b_{3}, \ldots$ etc. are the coefficients of normal 4-pole, 6-pole, ... component, $a_{1}, a_{2}$, $a_{3}, \ldots$ etc. are those of skew 4-pole, 6-pole, ... component, and $\mathrm{x}$ is the distance form the center of the aperture.

A harmonic coil with a diameter of $1.0 "$ and a length of $1^{\prime}$ (i.e. a Morgan coil ${ }^{4}$ ) was made and put inside a warm bore of 1.08" diameter. To eliminate toggling, a couple of rings were installed on the coil and the shaft to fit the inside diameter of the warm bore.

\subsection{Two-Dimentional Region}

\subsubsection{D.C. Measurement}

Various harmonic components measured using the DC mode are given in Figs. 16, 17, 18 and 19. The quadrupole component at $Y=0.5^{\prime \prime}$ is about $0.4 \%$ of the dipole field. No hysteresis behavior was observed, which can be expected from symmetry. A small remanent 4-pole component ( I.5 Gauss) exists (Fig. 16).

As for the 6-pole component, the value at $r=0.5^{\prime \prime}$ is roughly $0.1 \%$ of the dipole component. The hysteresis behavior can clearly be seen in Fig. 17. To confirm this behavior, the field was ramped up and down several times and the variation in the 6-pole component was measured. Fig. 18 shows a minor hysteresis loop. The remanent 6-pole component is about 1.5 Gauss, which corresponds to about $25 \%$ of the remanent dipole component. This hysteresis behavior is caused by the magnetization effect of a superconductor. Both the 8-pole and 10-pole components are less than $0.01 \%$ of the dipole at $Y=0.5^{\prime \prime}$ as is shown in Fig. 19. The above data, which are described in polar coordinates, are converted to Cartesian coordinates after the phase correction 
for the instrumental angle difference between the 2-pole and higher order pole coils. The harmonic coefficients expressed in Cartesian coordinates are shown in Fig. 20 as a function of the dipole field. The deviation from linearity of $b_{1}$ and $b_{2}$ below $10 \mathrm{kG}$ may be due to magnetization of superconductor. The increase of $b_{1}$ above $25 \mathrm{kG}$ may be due to coil movement relative to iron lamination or coil deformation.

Typical reconstructed field shape is shown in Fig. 21. This shape was roughly confirmed by a Hall probe. The normal quadrupole component is quite large, which is attributed to construction errors in the magnet, for example the miscentering of coil and iron. More careful assembly and winding could decrease the quadrupole component.

\subsubsection{Pulsed Measurement}

To investigate the effect due to the eddy currents between filaments or strands and arising from stainless parts, the harmonic components are measured using the pulsed mode. The results are given in Figs. 22 and 23.

The 4-pole component in this mode is the same as that in the D.C. mode. The pulsed 6-pole component showed an increase to about 2 Gauss in the difference between increasing and decreasing field, but the ramp rate dependence is rather weak. 8.2 End Effect

To know how the field is distributed at the ends the harmonic coil was positioned to cover the entire end region, and the 6-pole component was measured in the D.C. mode. The results are given in Table 1 , where data are for a one foot length of coil. The 6-pole term is considerably larger at the end region 
but the integrated 6-pole component over the central region is still larger than that over the end region.

Table 1

End Effect

6-pole Component with I' Harmonic Coil

\begin{tabular}{|c|c|c|}
\hline $\begin{array}{c}\text { dipole field } \\
(\mathrm{kG})\end{array}$ & $\begin{array}{c}\text { center region } \\
\text { Gauss }\end{array}$ & $\begin{array}{c}\text { end region } \\
\text { Gauss }\end{array}$ \\
\hline 5 & 6.7 & 16.3 \\
\hline 10 & 12.5 & 31.1 \\
\hline 15 & 23.9 & 63 \\
\hline
\end{tabular}




\section{$\underline{\text { References }}$}

1. R. Yamada et al, Fermilab Internal Report TM-638, December 30, 1975, "AC Loss Test of Di0-3 Magnet".

2. M. Kuchnir, TM-645, February 17, 1976, "Heat Leak Measurements on a D-10'Cryostat"

3. R. Yamada et al, TM-598, July 1975, "Superconducting Wire Test at Fermilab"

4. G.H. Morgan, Proceedings of the Fourth International Conf. on Magnet Technology, p. 787, "Stationary Coil for Measuring the Harmonics in Pulsed Transport Magnets" 


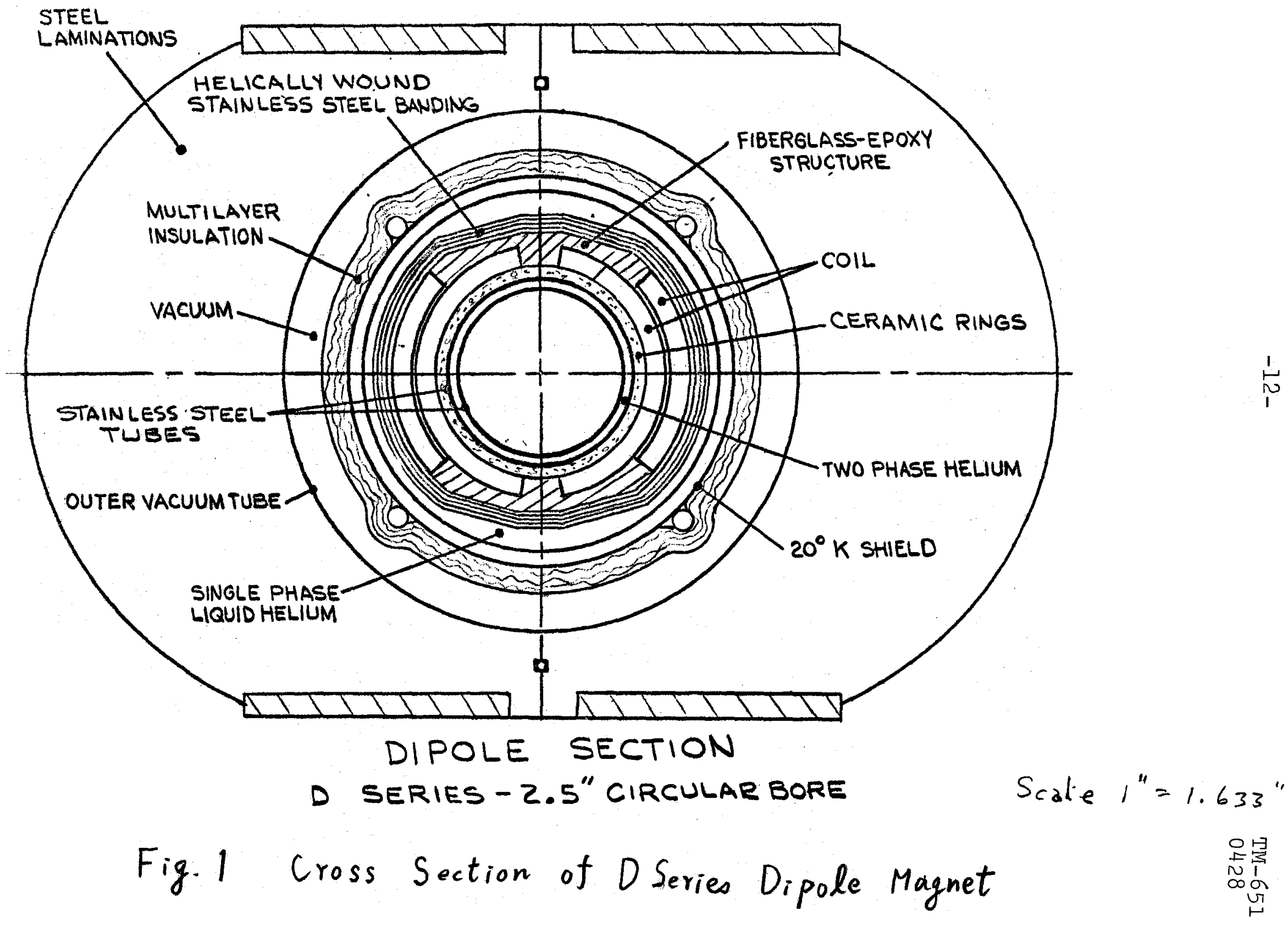




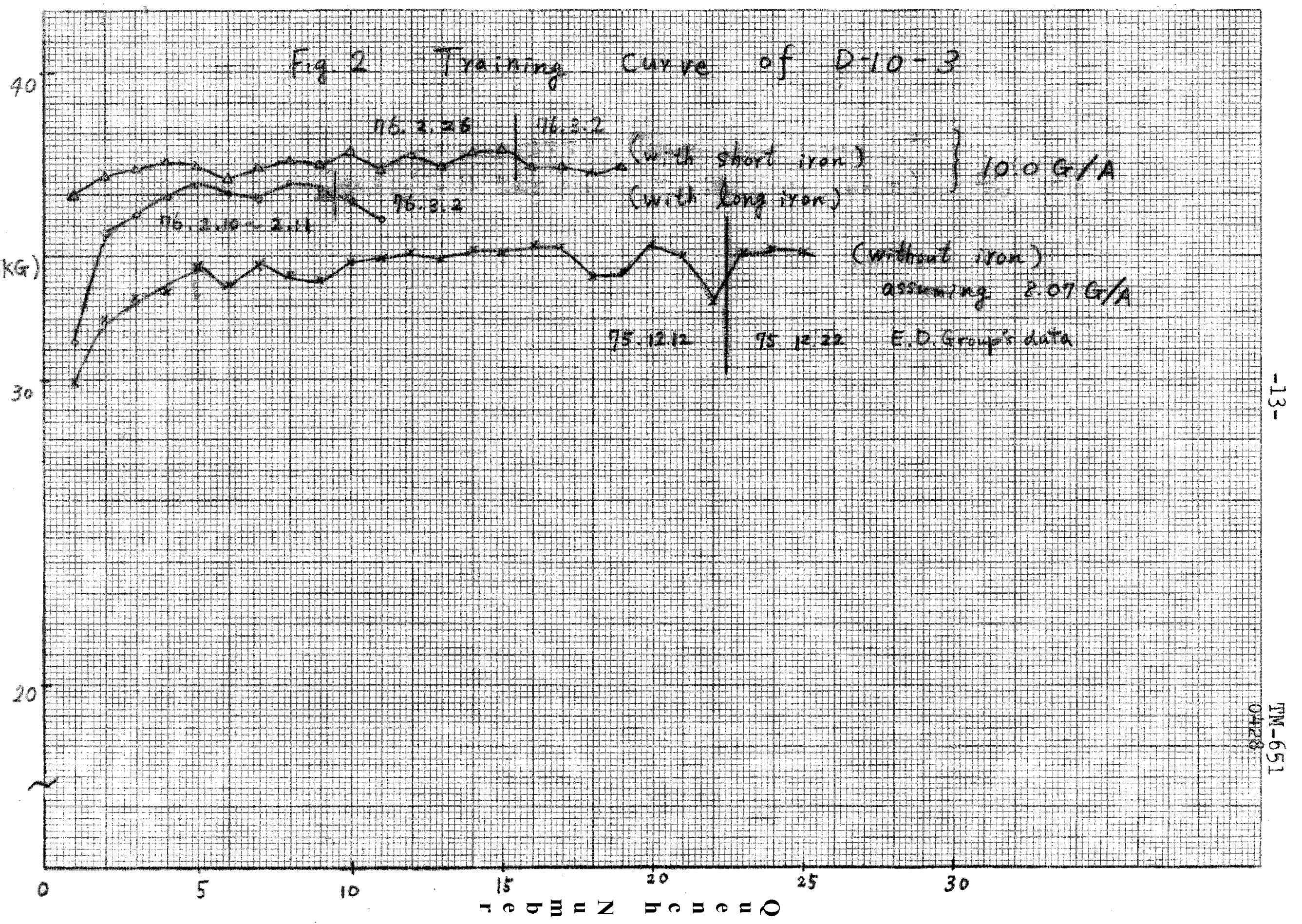


$-14-$

TM-651

5,0000

\section{$0-10-3$ Magnet \\ iMCA $M$ thand}

(Amp) unsoldered cable)

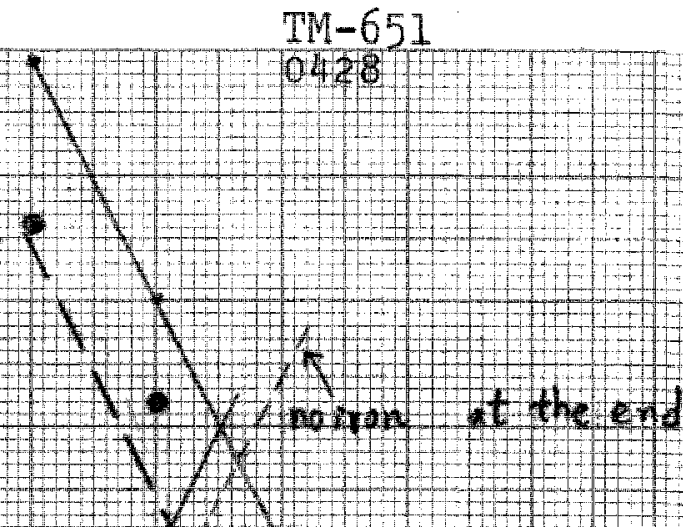

4000

3000

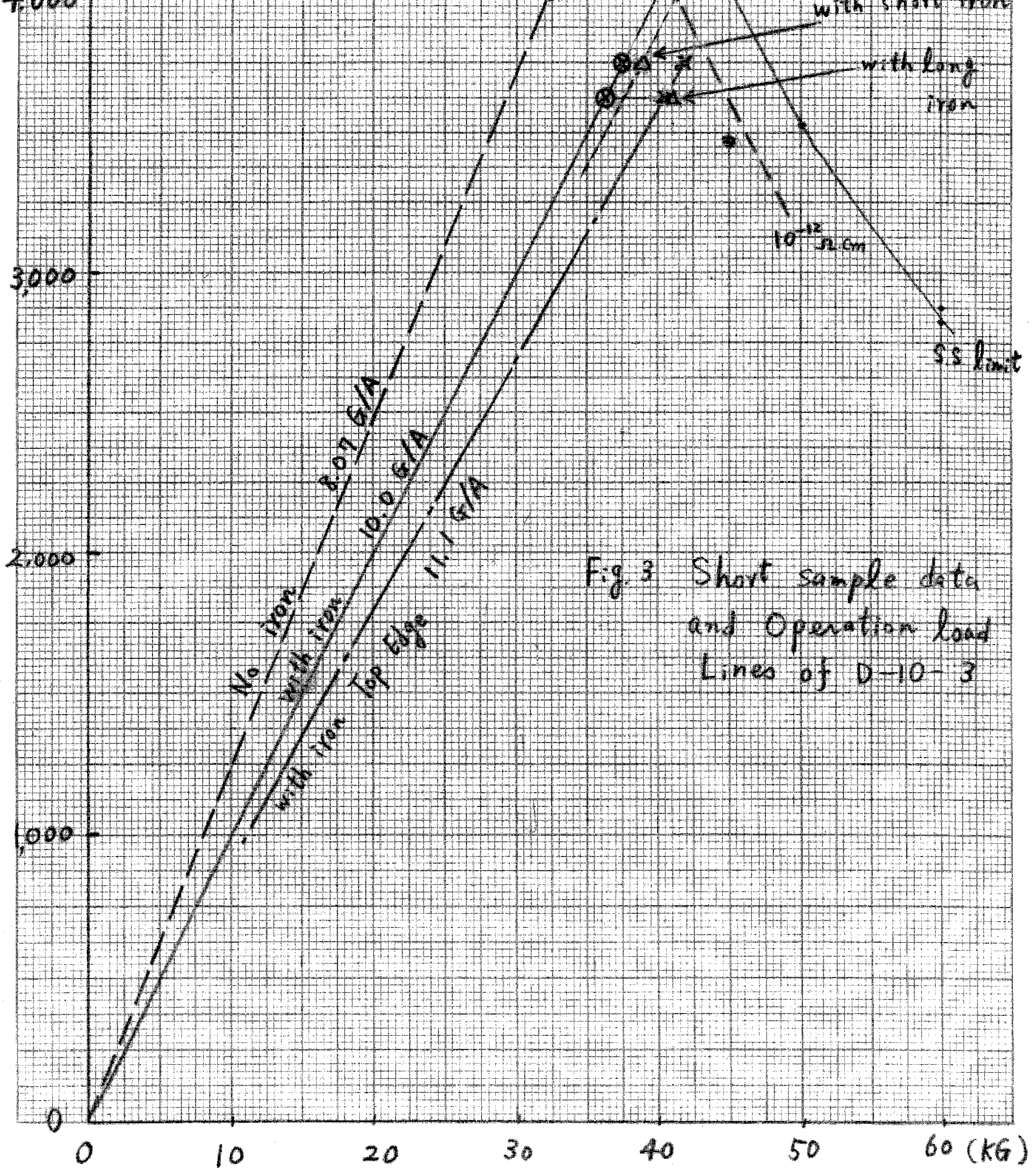

2,000

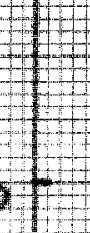




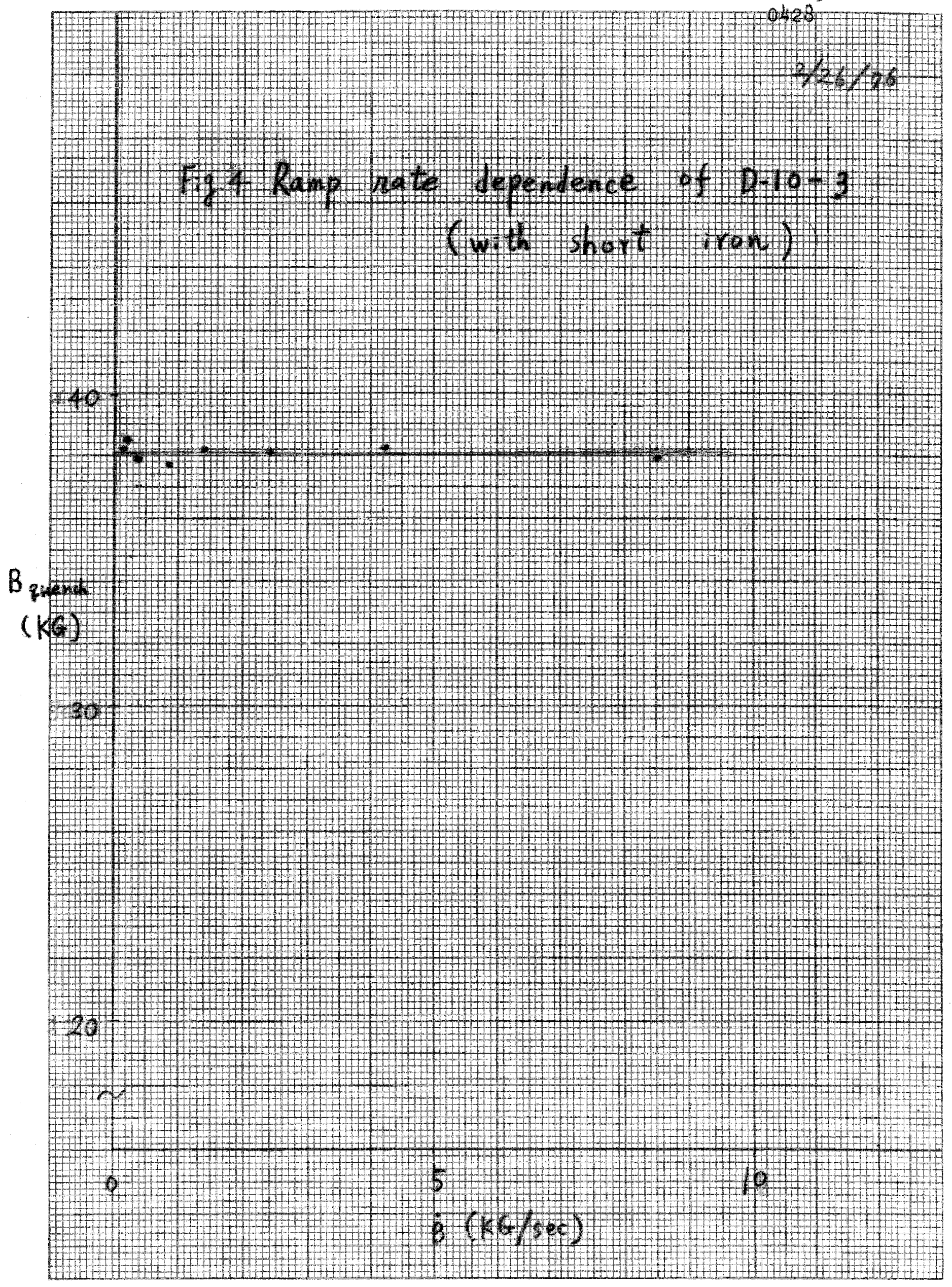


Fig 5 lemperathre dependence of aumat Carrent D-10-3 with short Iran

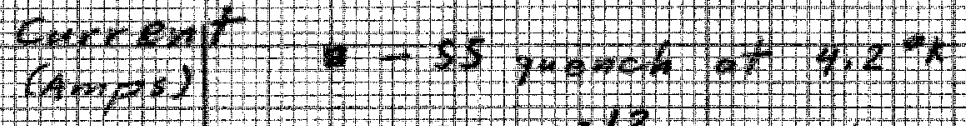

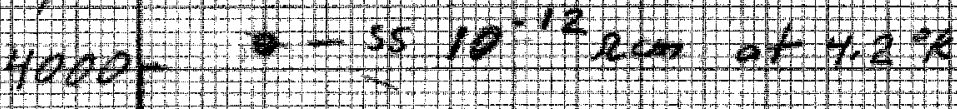

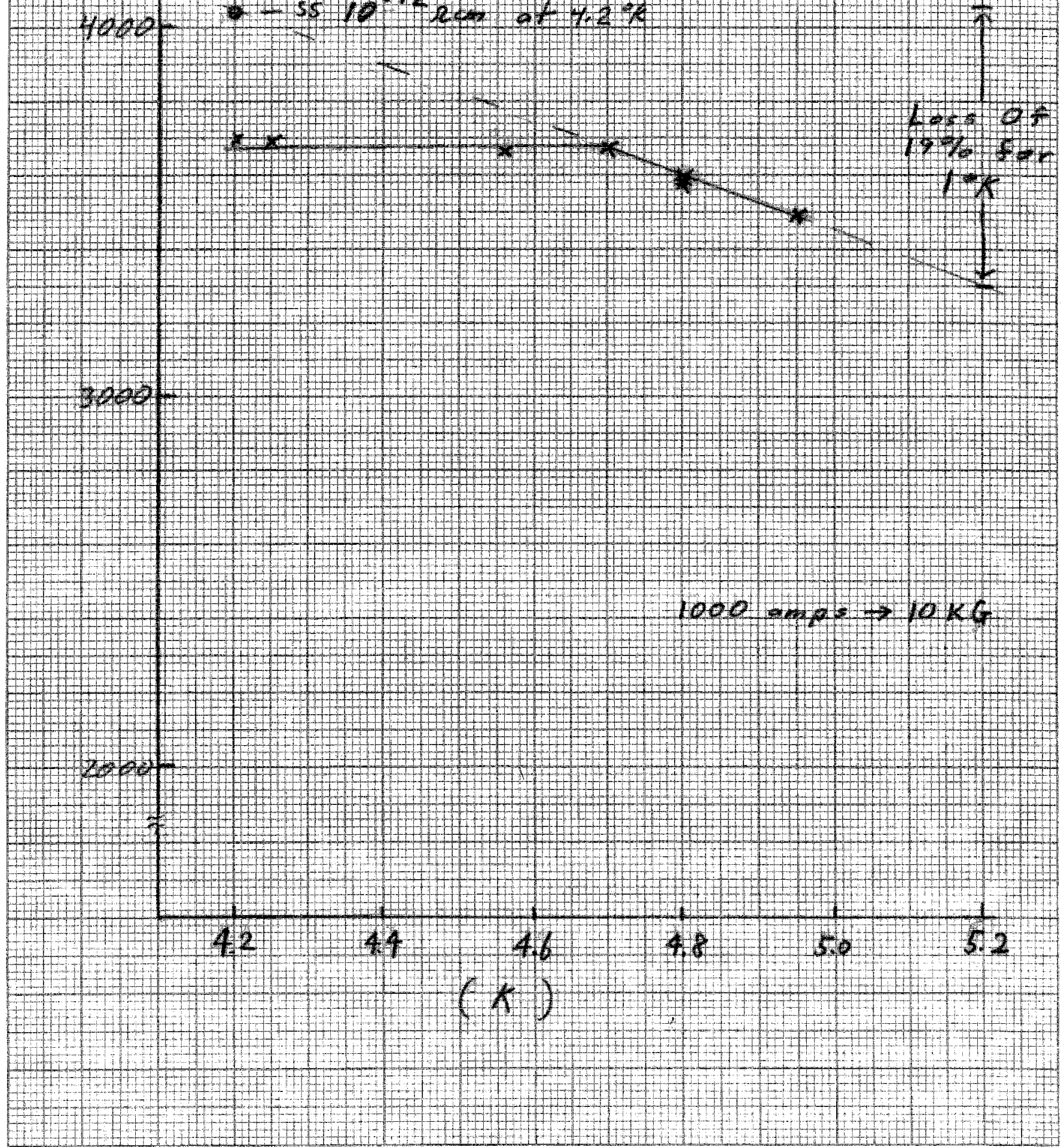




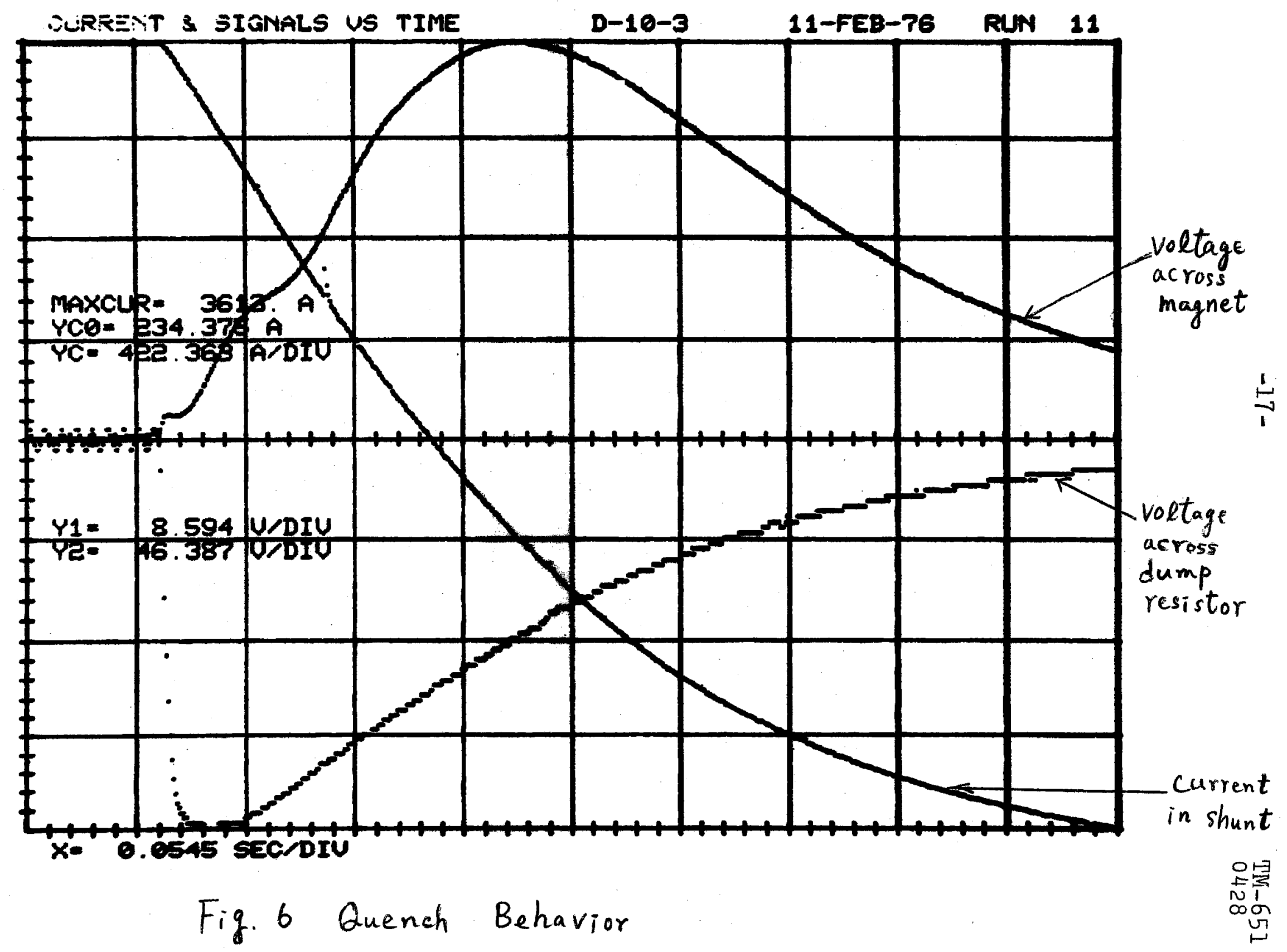




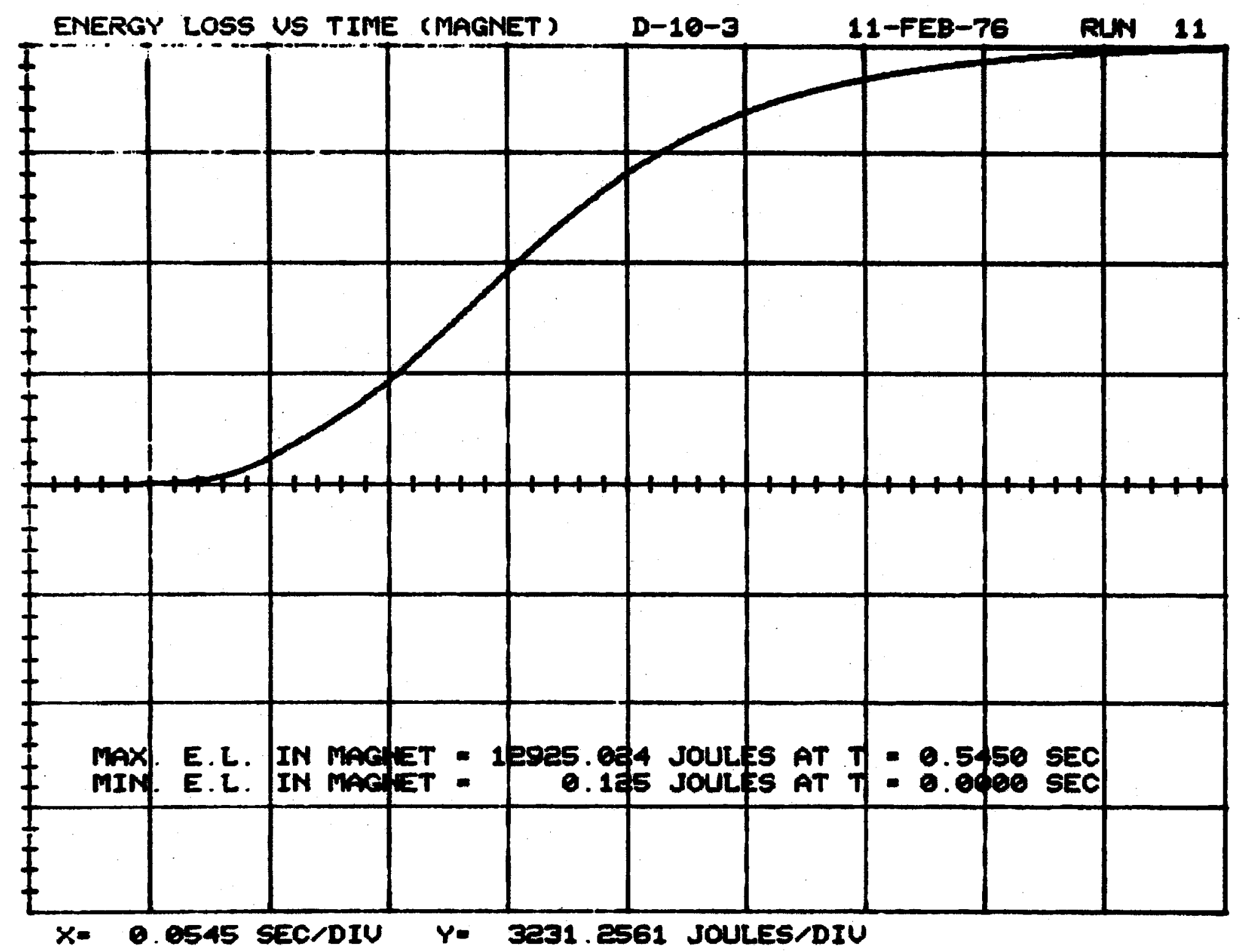

Fig. 7 Energy Loss in Magnet 

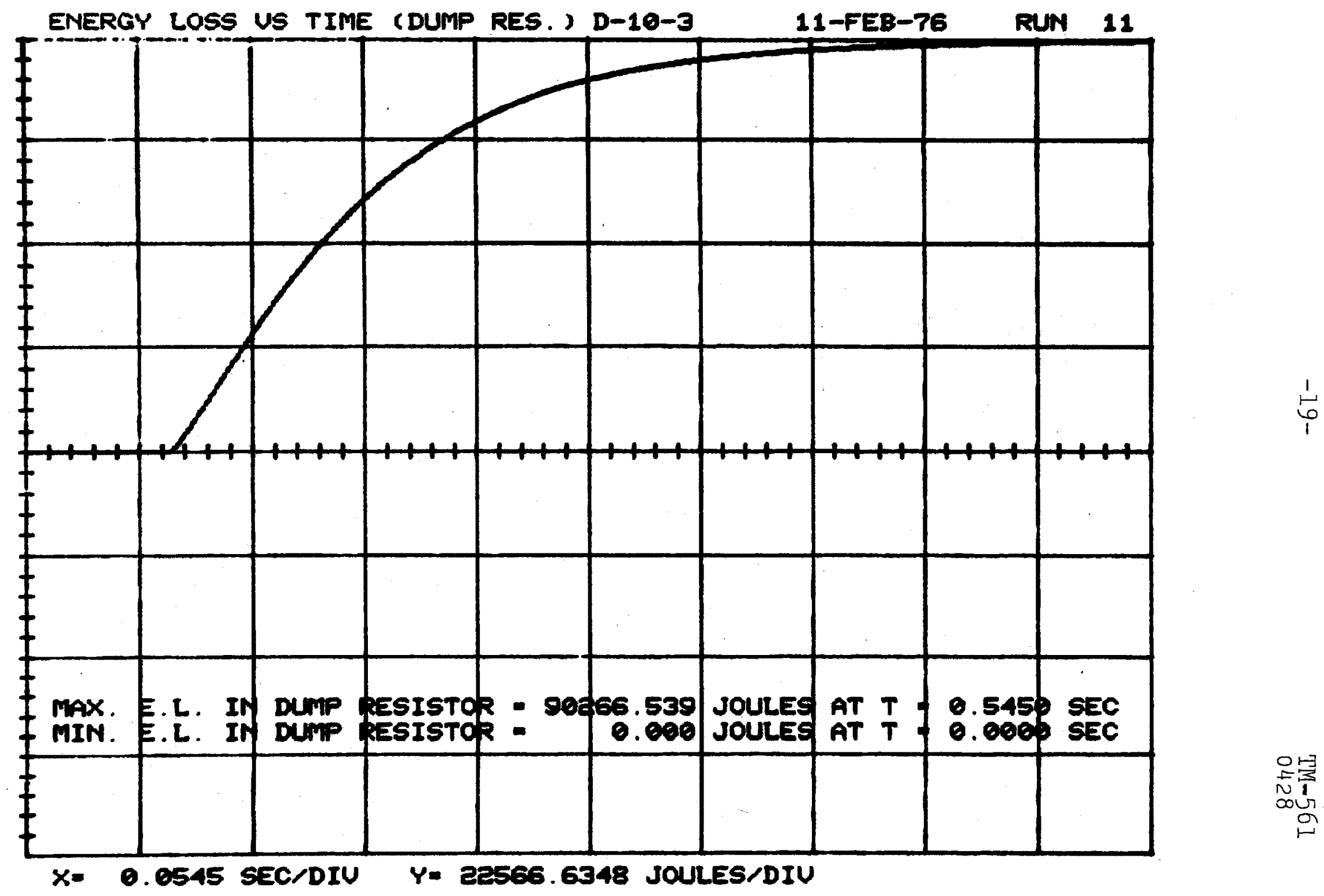

Fig. 8 Energy Loss in Dump Resistor 


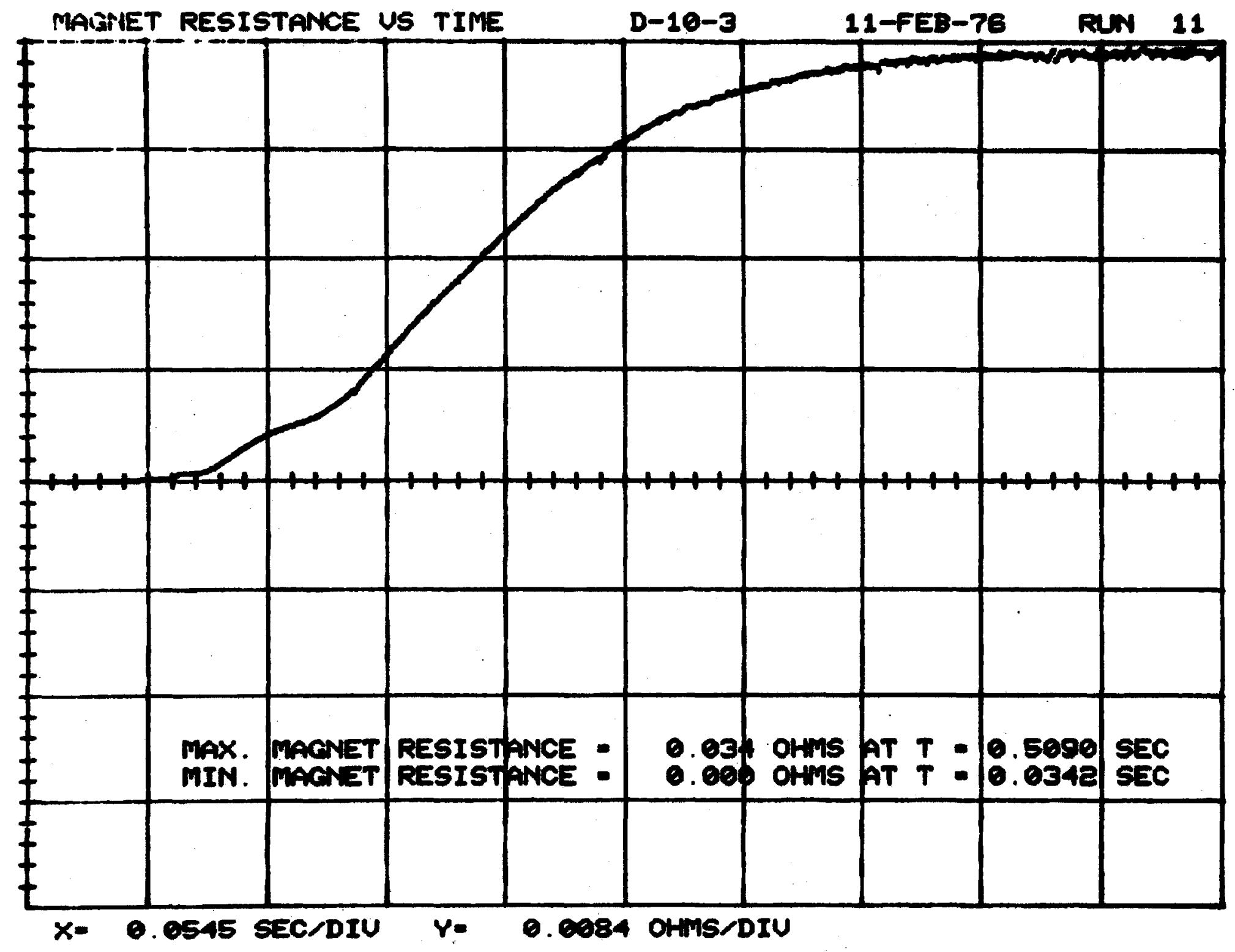

Fig. 9 Magnet Resistance 
Iq10

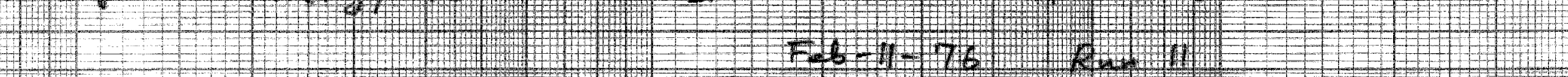

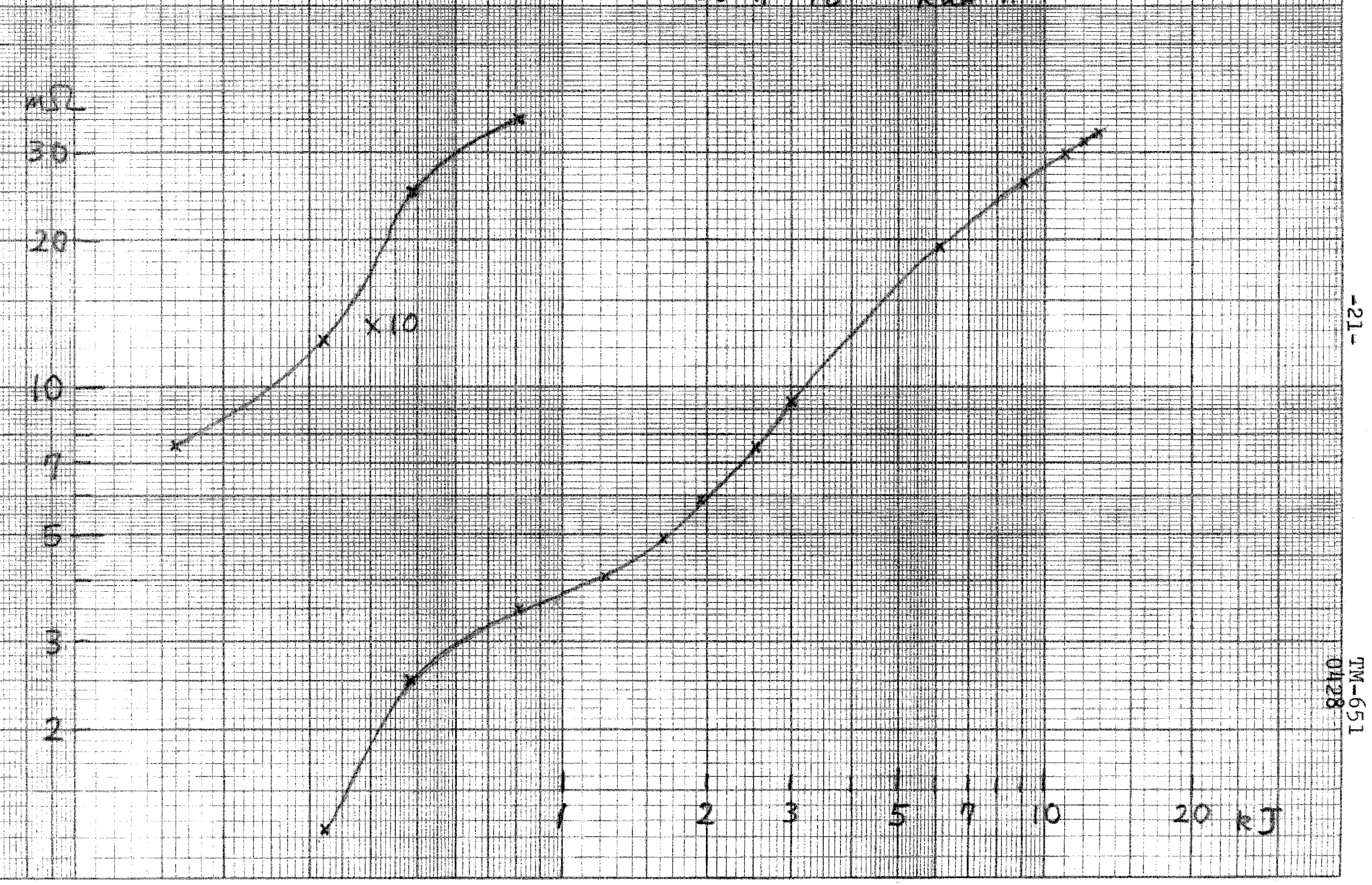




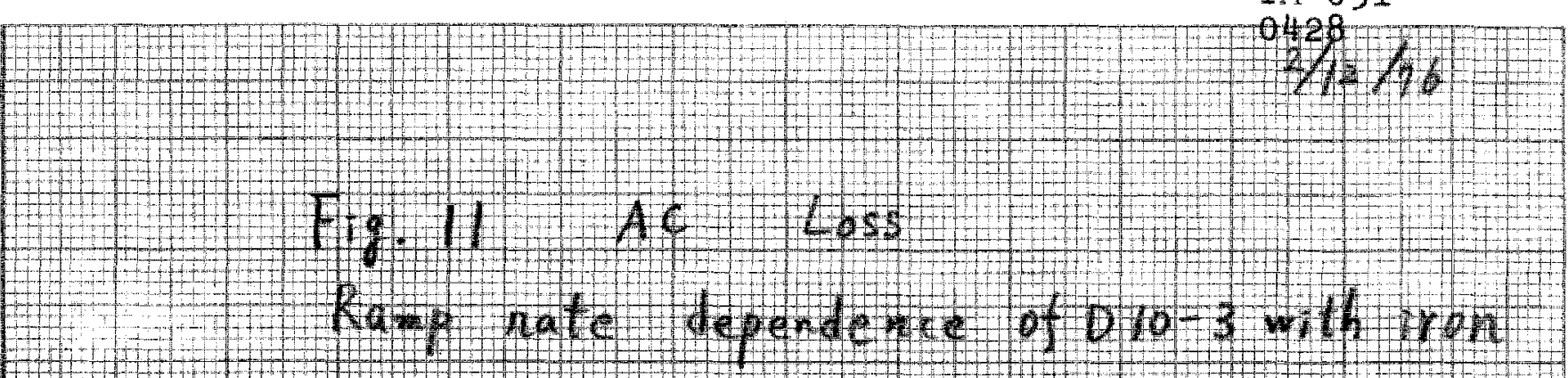

3 $+4+1+1+$

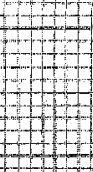

100

50
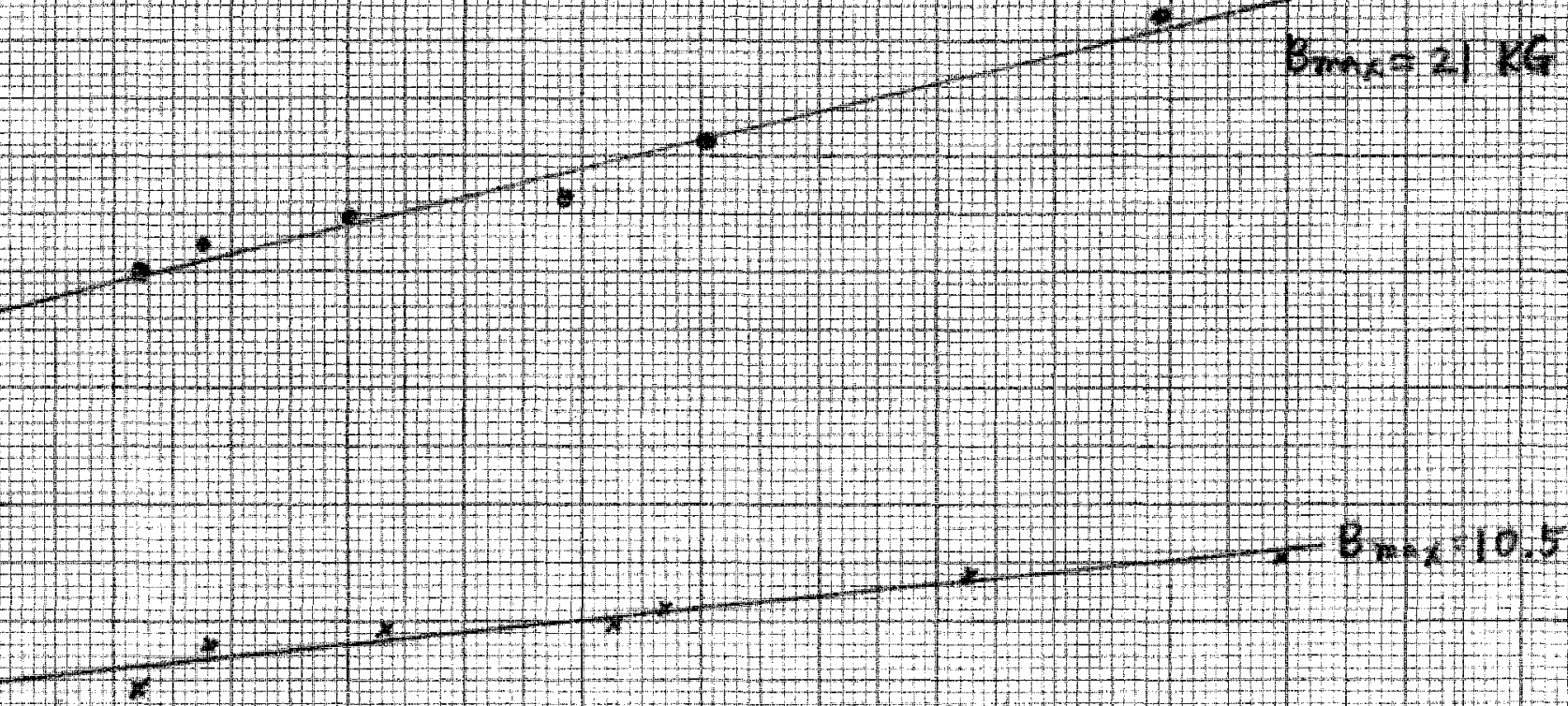

B.man $10.5 \mathrm{KG}$ 
Fig.12, $A C_{6, \text { L Loss }}$

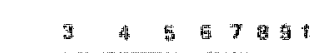
装 3

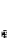

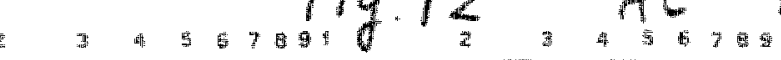

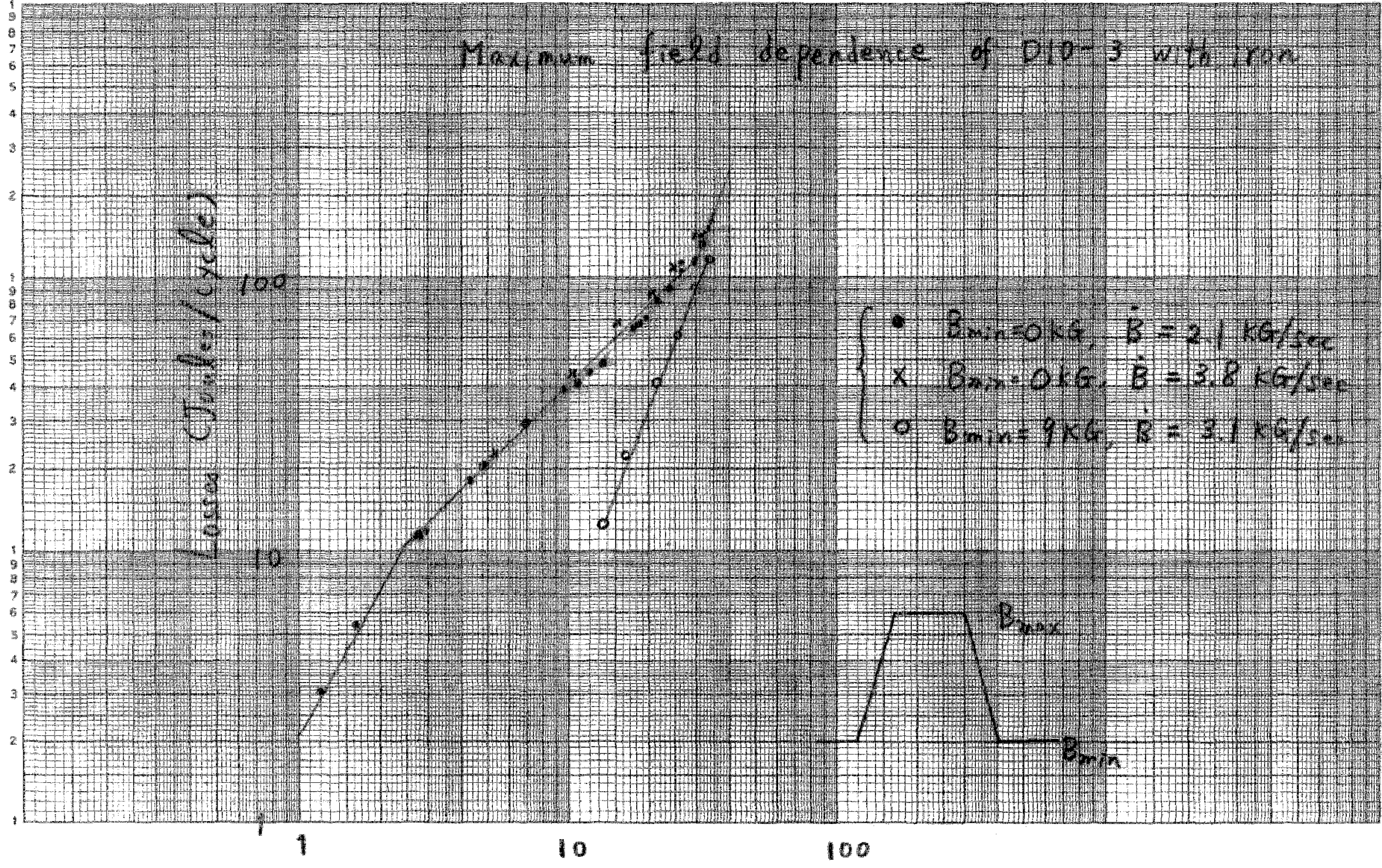




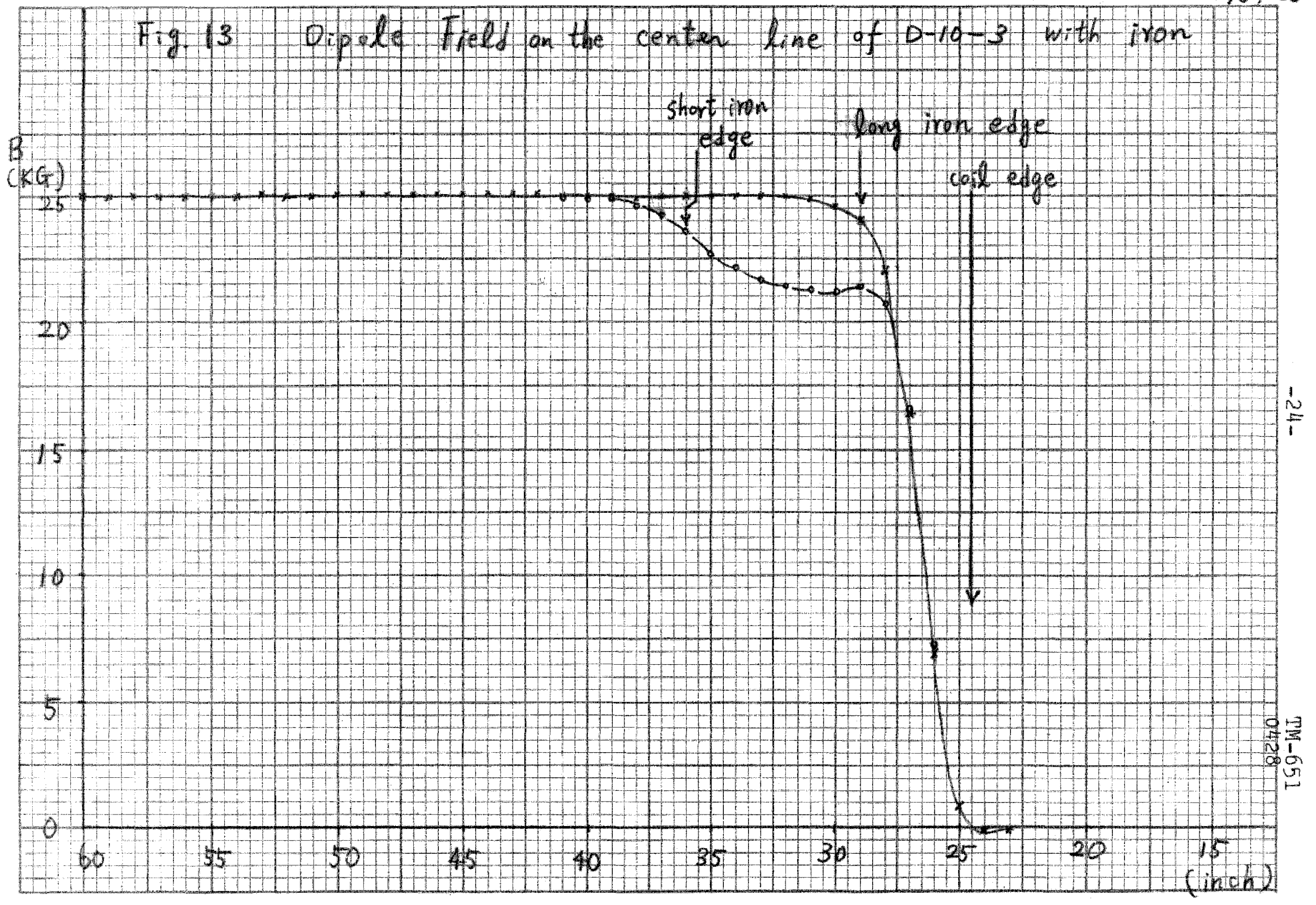


Fig. 14 Remanent Field on Center line ( D-10-3 with longeron) 2/12/76

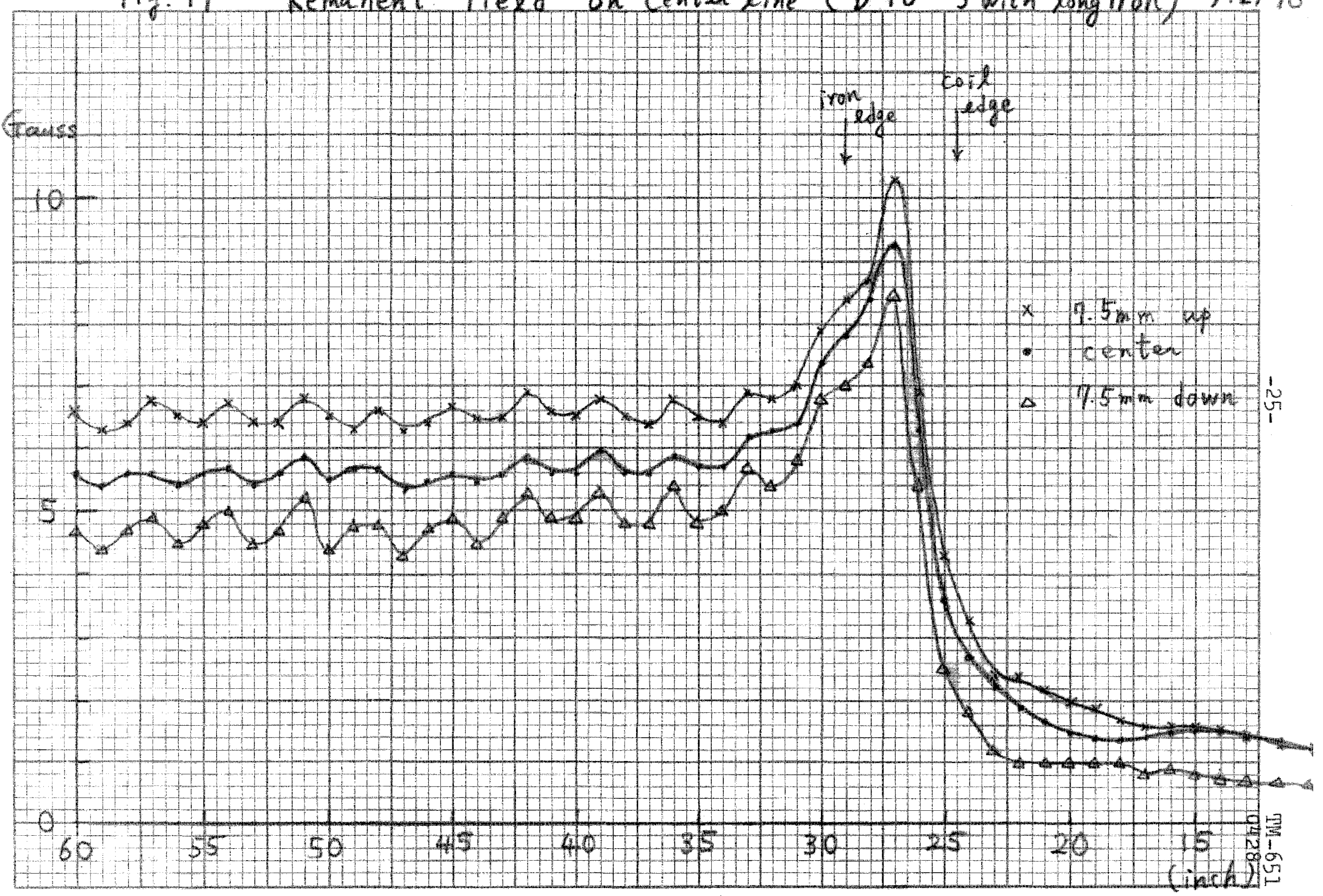


Fig. 15 Block Diagram of Harmonic Analysis Equipment

$$
\text { CAMAC }
$$

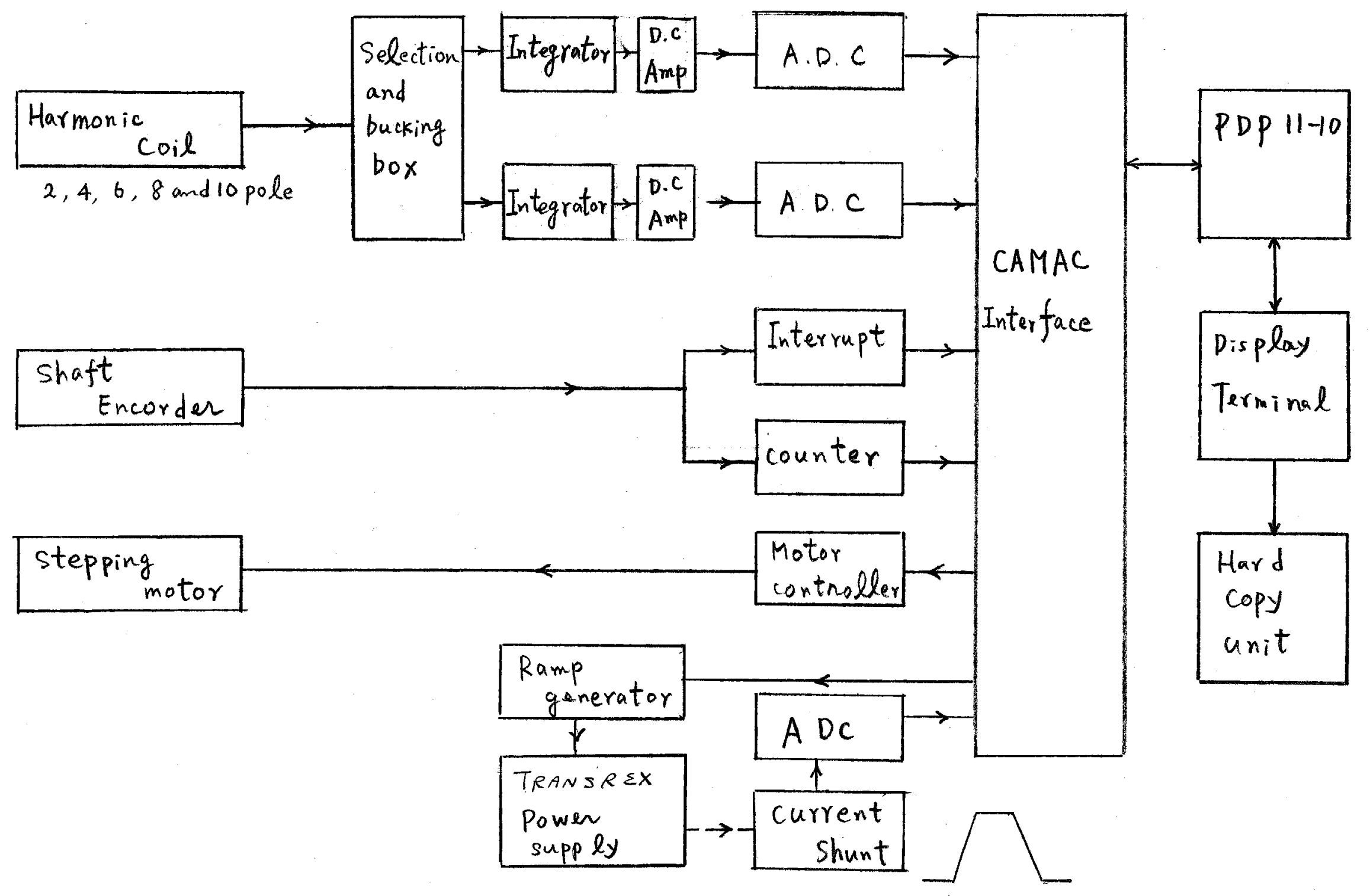


$-27=$

TM-651 $\quad 2 / 79 / 26$

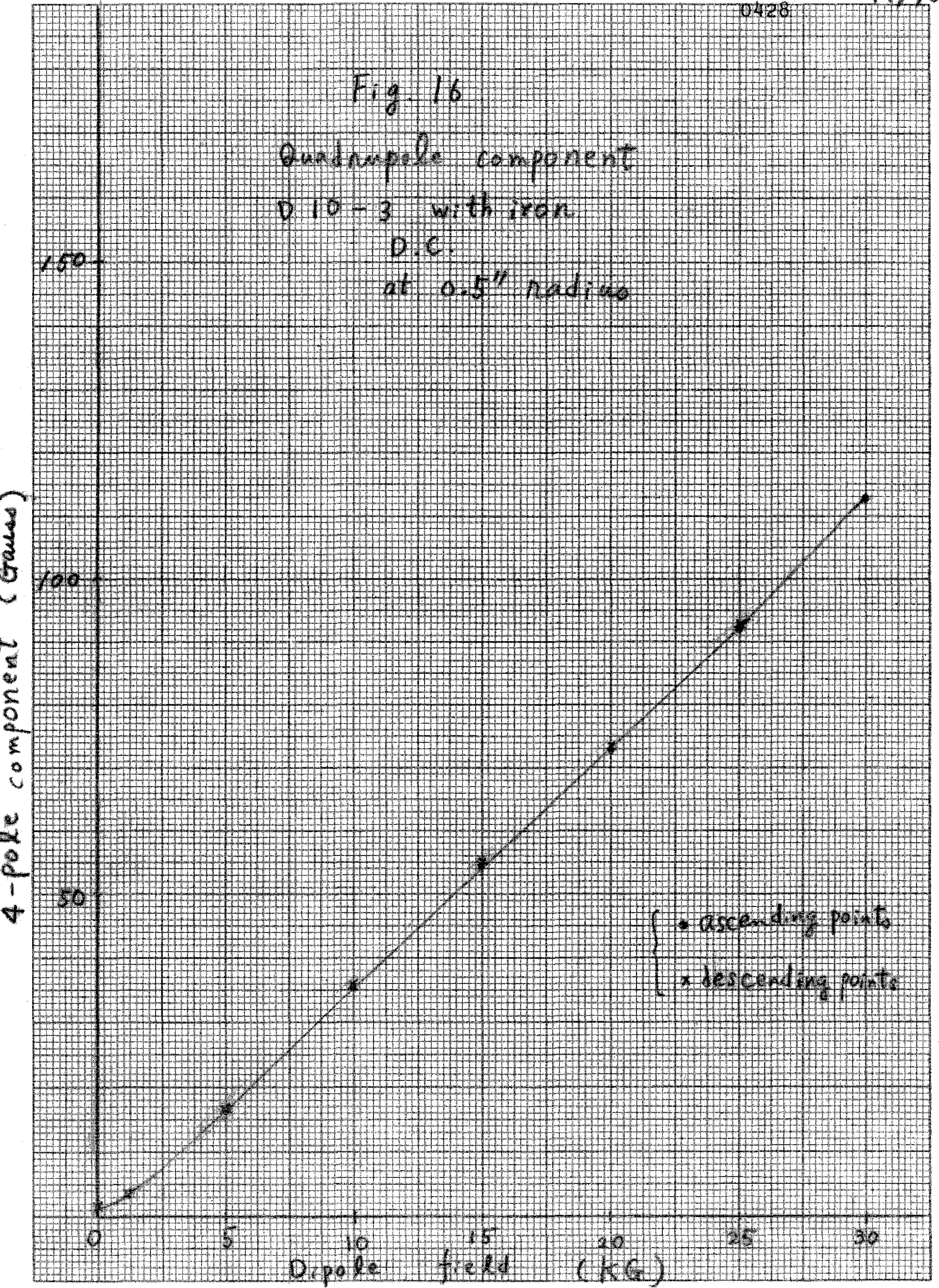




$$
-28-
$$

TM-65I $2 / 10 / 3$

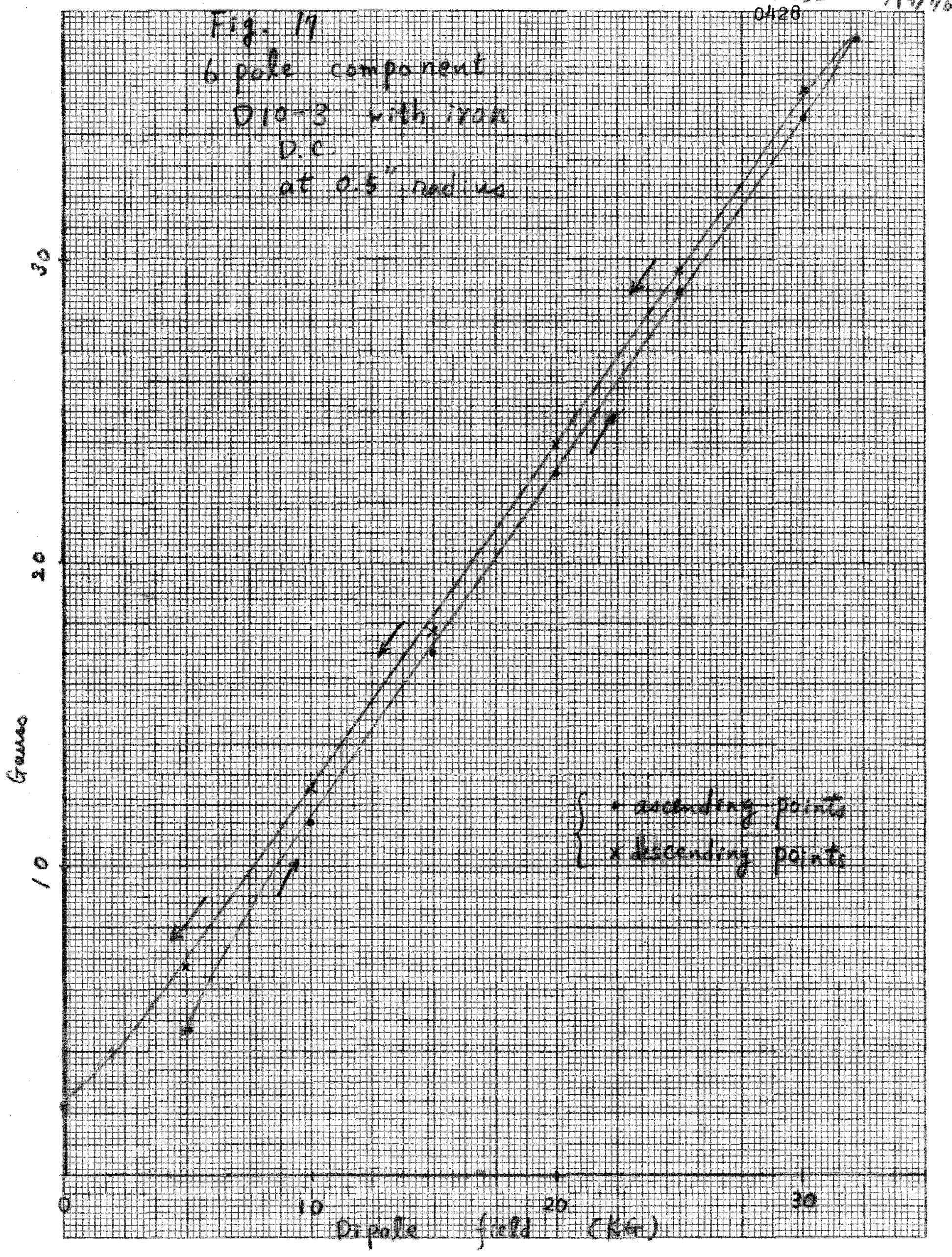


$-29-\quad T M-651 \quad 2 / 19 / 96$

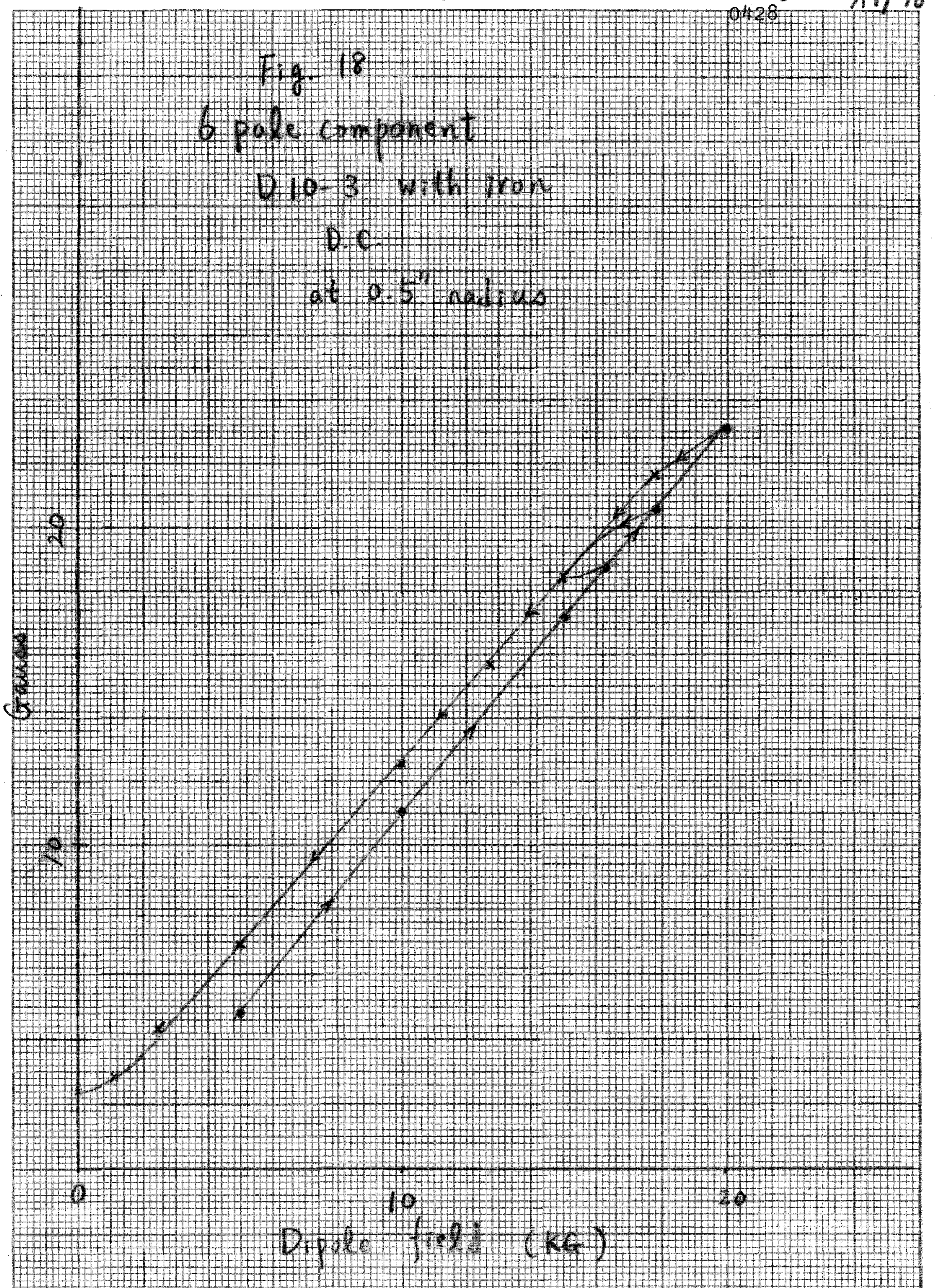




$$
-30-\quad 29-651 \quad 2 / 22 / 46
$$

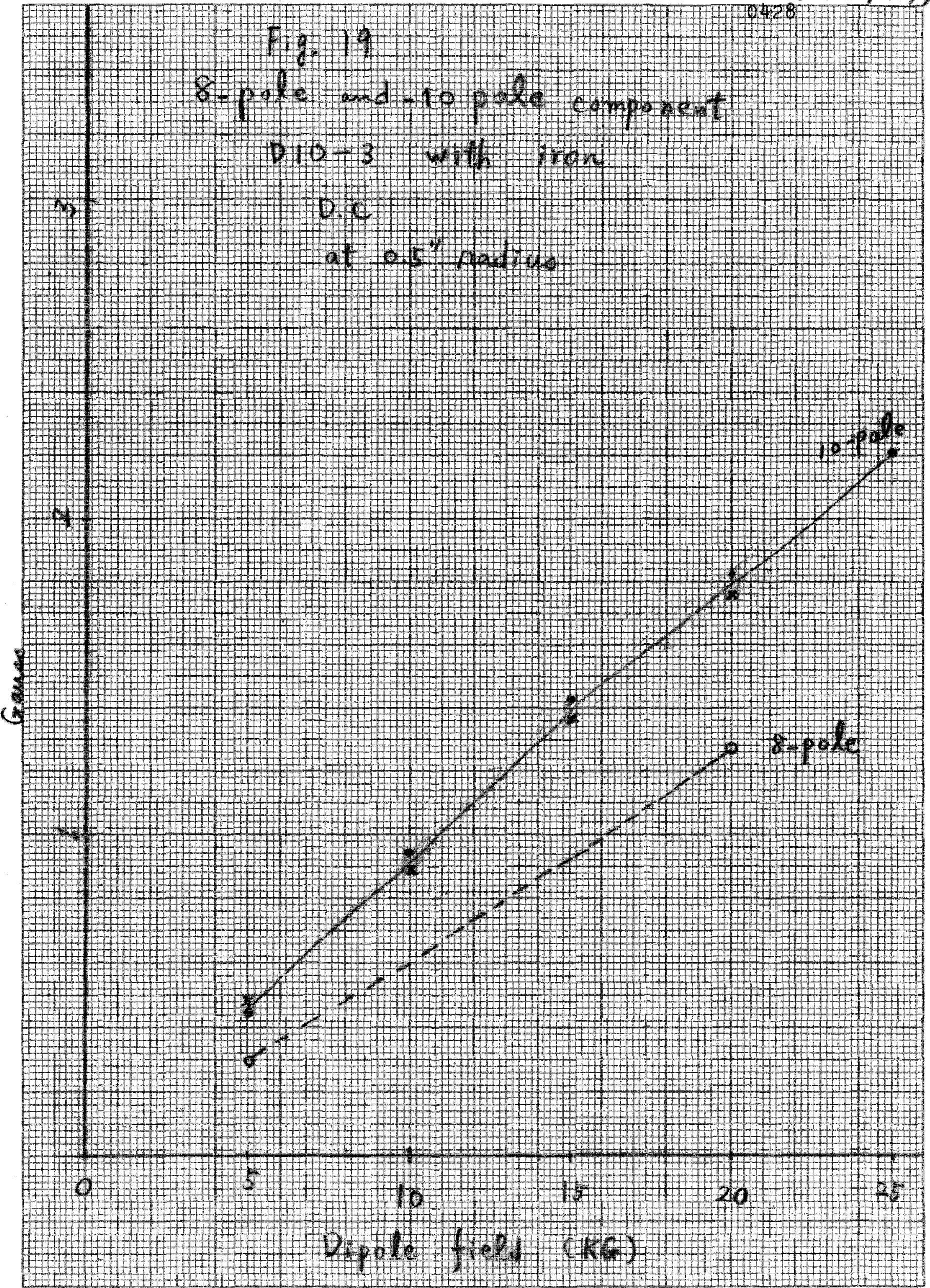


$-31-$

TM-65I

lig 20 Variotion of aN-pole coetficient?

30

25

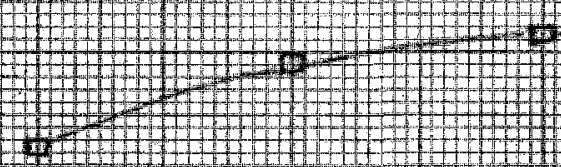

10

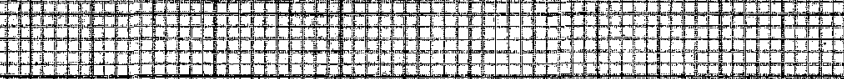

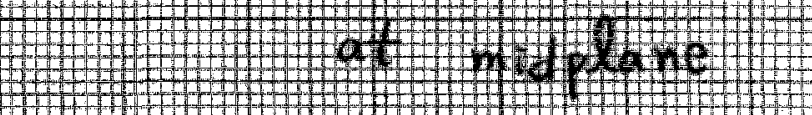

B. $(x)=(1)+(x+2)$

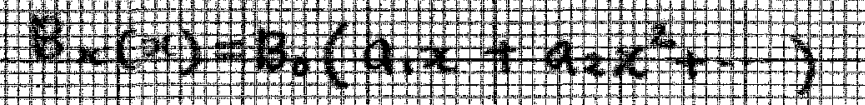

${ }^{7}+\frac{5}{4}$

\begin{tabular}{ll} 
L \\
\hline
\end{tabular} 
$-32-\quad T M-651$

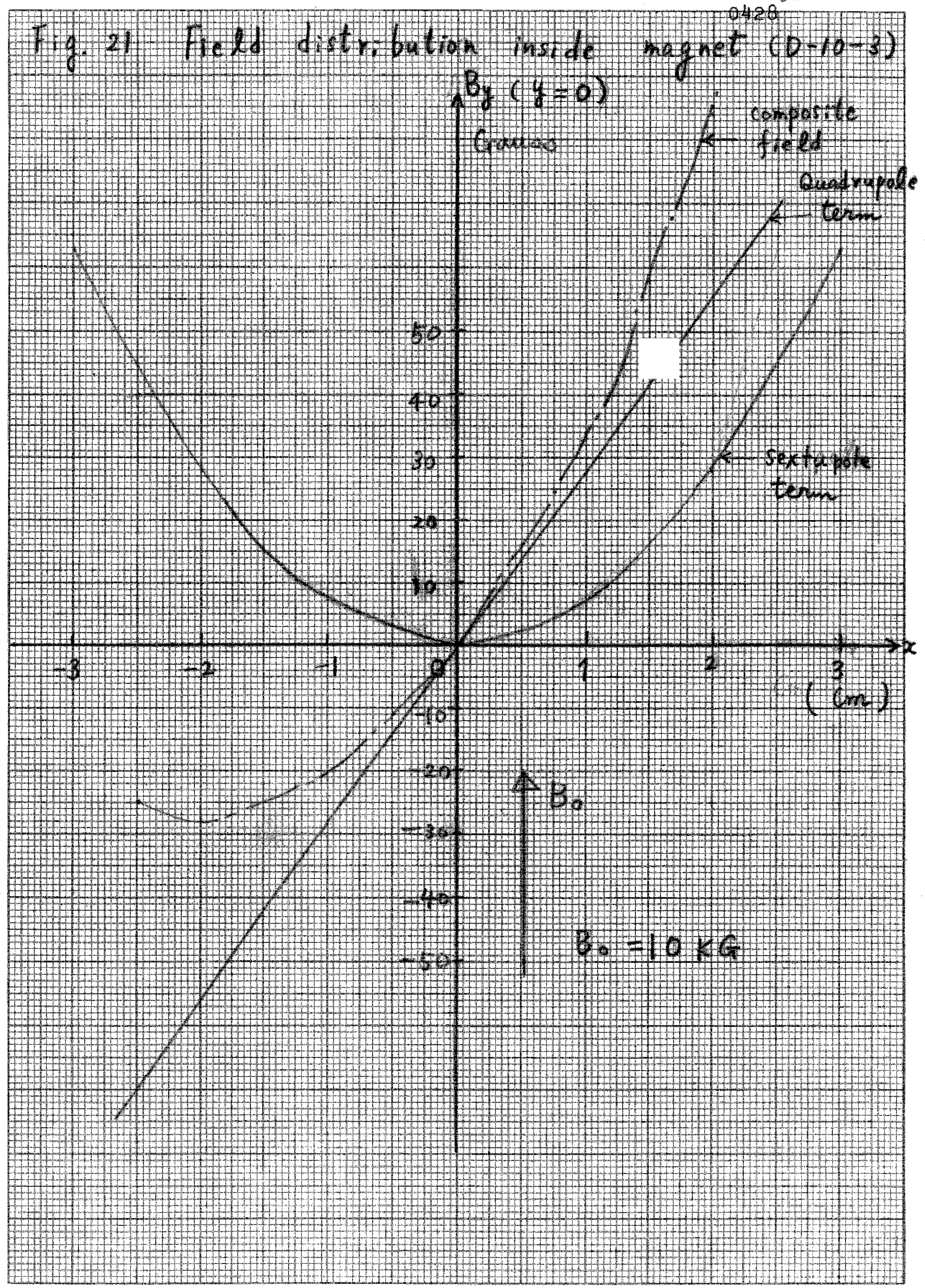


$-34-$

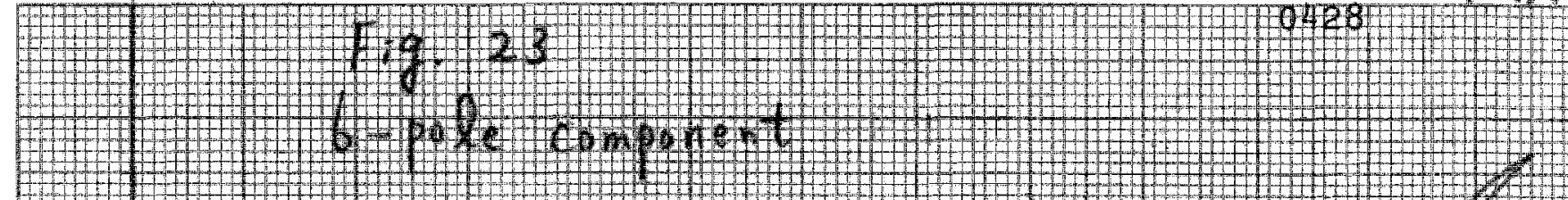

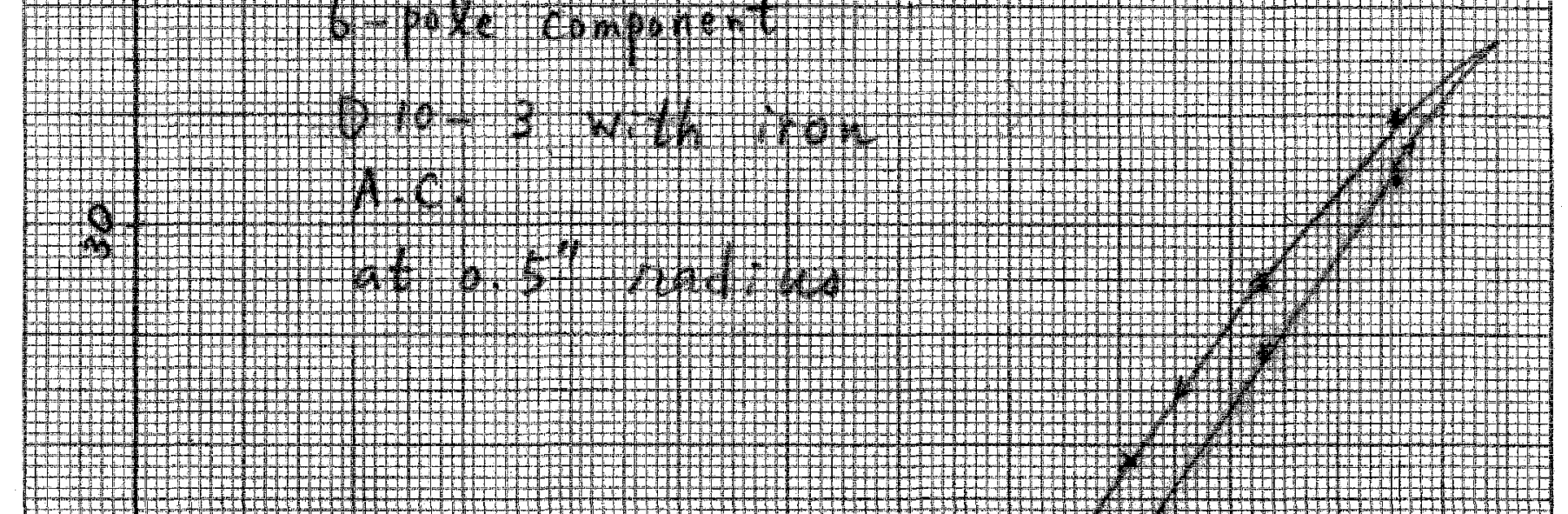
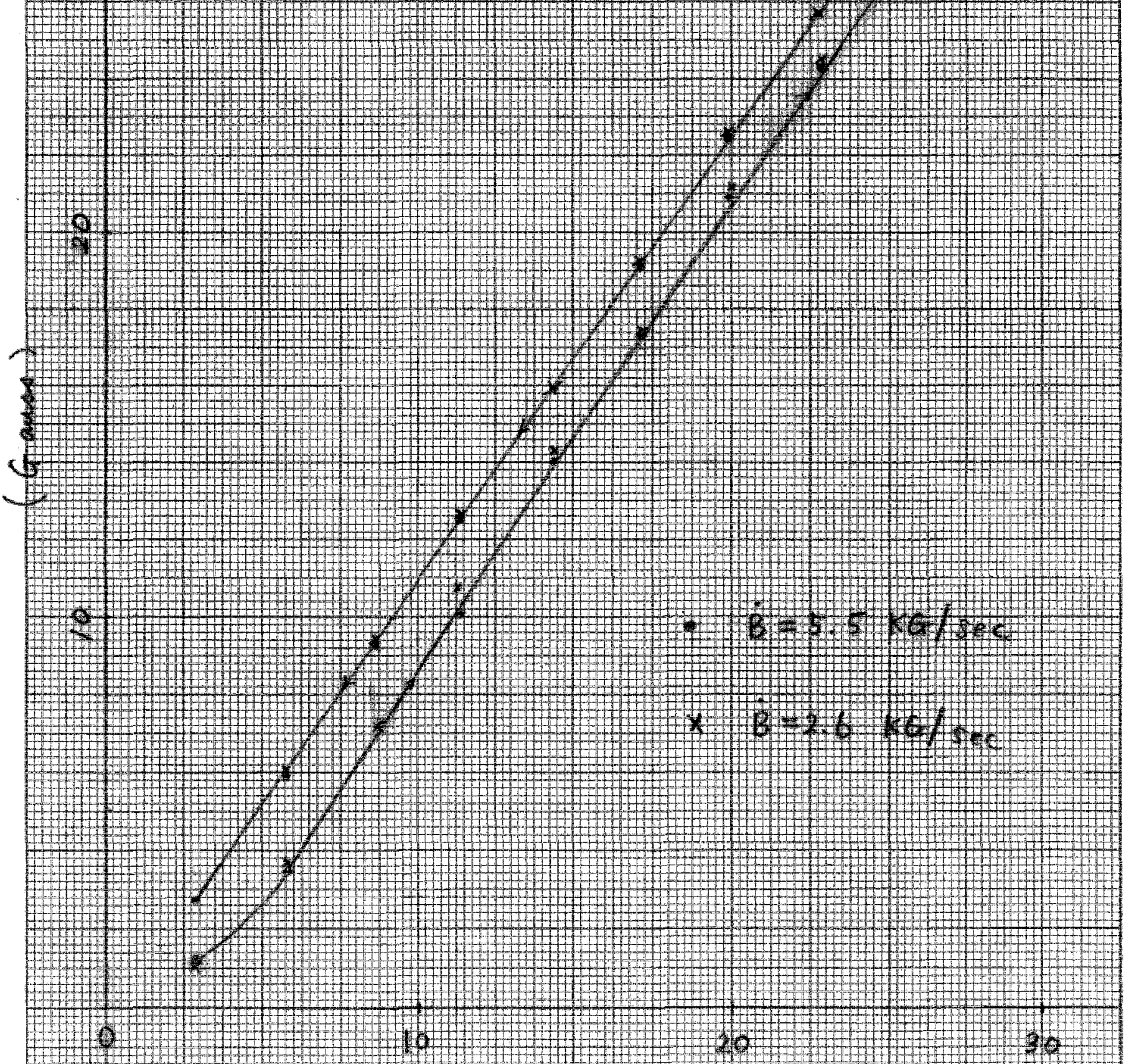

Opole $(p e)(k G)$ 